OPEN ACCESS

Edited by:

Roland Wohlgemuth,

Łódź University of Technology, Poland

Reviewed by:

Yehong Du,

Chongqing Medical University, China

Thomas Pulinilkunnil,

Dalhousie University, Canada

${ }^{*}$ Correspondence:

Yong-ming Yao

c_ff@sina.com

Xiao-hui Du

duxiaohui301@sina.com

Chao Ren

rc198@sina.com

${ }^{\dagger}$ These authors have contributed equally to this work

Specialty section:

This article was submitted to Molecular and Cellular Pathology,

a section of the journal

Frontiers in Cell and Developmental

Biology

Received: 14 February 2021

Accepted: 28 July 2021

Published: 13 August 2021

Citation:

Zhu S-y, Yao R-q, Li Y-x

Zhao P-y, Ren C, DuX-h and Yao Y-m

(2021) The Role and Regulatory Mechanism of Transcription Factor EB

in Health and Diseases.

Front. Cell Dev. Biol. 9:667750.

doi: 10.3389/fcell.2021.667750

\section{The Role and Regulatory Mechanism of Transcription Factor EB in Health and Diseases}

\author{
Sheng-yu Zhu',2,3t, Ren-qi Yao ${ }^{1,4 t}$, Yu-xuan Li², Peng-yue Zhao'2, Chao Ren ${ }^{1 *}$, \\ Xiao-hui Du ${ }^{2 *}$ and Yong-ming Yao ${ }^{1 *}$ \\ ${ }^{1}$ Medical Innovation Research Division, Translational Medicine Research Center and Fourth Medical Center of the Chinese \\ PLA General Hospital, Beijing, China, ${ }^{2}$ Department of General Surgery, First Medical Center of Chinese PLA General \\ Hospital, Beijing, China, ${ }^{3}$ School of Medicine, Nankai University, Tianjin, China, ${ }^{4}$ Department of Burn Surgery, Changhai \\ Hospital, Naval Medical University, Shanghai, China
}

Transcription factor EB (TFEB) is a member of the microphthalmia-associated transcription factor/transcription factor $\mathrm{E}$ (MiTF/TFE) family and critically involved in the maintenance of structural integrity and functional balance of multiple cells. In this review, we described the effects of post-transcriptional modifications, including phosphorylation, acetylation, SUMOylation, and ubiquitination, on the subcellular localization and activation of TFEB. The activated TFEB enters into the nucleus and induces the expressions of targeted genes. We then presented the role of TFEB in the biosynthesis of multiple organelles, completion of lysosome-autophagy pathway, metabolism regulation, immune, and inflammatory responses. This review compiles existing knowledge in the understanding of TFEB regulation and function, covering its essential role in response to cellular stress. We further elaborated the involvement of TFEB dysregulation in the pathophysiological process of various diseases, such as the catabolic hyperactivity in tumors, the accumulation of abnormal aggregates in neurodegenerative diseases, and the aberrant host responses in inflammatory diseases. In this review, multiple drugs have also been introduced, which enable regulating the translocation and activation of TFEB, showing beneficial effects in mitigating various disease models. Therefore, TFEB might serve as a potential therapeutic target for human diseases. The limitation of this review is that the mechanism of TFEB-related human diseases mainly focuses on its association with lysosome and autophagy, which needs deep description of other mechanism in diseases progression after getting more advanced information.

Keywords: transcription factor EB, autophagy, tumor, inflammation, organelles

\section{INTRODUCTION}

Transcription factor EB (TFEB) belongs to the microphthalmia-associated transcription factor/transcription factor $\mathrm{E}$ (MiTF/TFE) family, which transcriptionally regulates the expression of multiple genes in response to various stimuli (Napolitano and Ballabio, 2016). Study by Puertollano et al. (2018) showed that the cytoplasmic-nucleus shuttling of TFEB was largely regulated by 
transitions between phosphorylation and dephosphorylation. In normal condition, TFEB is mainly remained in the cytoplasm with inactivated form mediated by phosphorylation (Martina et al., 2012; Roczniak-Ferguson et al., 2012; Settembre et al., 2012). The activation and translocation of TFEB are sequentially initiated under exposure of cellular stress, such as starvation and lysosomal dysfunction (Martina et al., 2012; Roczniak-Ferguson et al., 2012; Medina et al., 2015). TFEB rapidly shuts down the activation of target genes through nuclear export upon nutrient refeeding (Li et al., 2018a; Napolitano et al., 2018). It has been identified that TFEB is critically involved in the maintenance of structural integrity and functional balance of multiple cells by promoting biosynthesis of lysosomes and mitochondria (Sardiello et al., 2009; Wang S. et al., 2020), which is favorable for the homeostasis of host immune response (Martini-Stoica et al., 2016; Brady et al., 2018b). Activation of TFEB is capable of upregulating the expression of multiple lysosomal proteins by identifying and combining the coordinating lysosomal expression and regulation (CLEAR) elements (Sardiello et al., 2009). By regulating the function and number of lysosomes, TFEB is essential for the completion of all types of autophagy, including macroautophagy, microautophagy, and chaperone mediated autophagy (CMA). Moreover, TFEB is involved in cargo recognition, formation of autophagosomes, and the fusion between autophagosomes and lysosomes (Settembre et al., 2011; Kim et al., 2018). Dysregulation of TFEB activity, however, was reportedly contributed to the development of various diseases, including catabolic hyperactivity in tumors, accumulation of abnormal aggregates in neurodegenerative diseases, and aberrant host responses in inflammatory diseases. Therefore, TFEB might serve as a potential therapeutic target for the treatment of human diseases. For example, the inhibition of TFEB is noted to enhance metabolic pressure or increase chemotherapy sensitivity for tumor cells, while upregulation of TFEB activity is capable of migrating oxidative stress and facilitating the clearance of inflammatory substances in inflammatory states. In terms of neurodegenerative diseases, the accumulation of pathogenic proteins can be alleviated by enhancing TFEB activity due to strong capacity in improving lysosomal function.

\section{THE MiTF/TFE FAMILY OF TRANSCRIPTION FACTORS}

In addition to TFEB, other transcription factors have been identified belonging to the MiTF/TFE family, including MiTF, transcription factor EC (TFEC), and transcription factor E3 (TFE3) (Napolitano and Ballabio, 2016; Slade and Pulinilkunnil, 2017). These factors share the similar domains for efficient transcription: the basic domain for DNA binding, and the helixloop-helix as well as leucine zipper (BHLH-LZ) domains which serve as the formation sites for homodimers or heterodimers in association with the other family members (Aksan and Goding, 1998). Of note, these transcription factors can also form dimers with themselves (Hemesath et al., 1994; Muhle-Goll et al., 1994), which are incapable to bind with other proteins containing BHLH-LZ domains due to unusual three-residue shift within the leucine zipper register (Pogenberg et al., 2012). Unlike other
BHLH-Zip transcription factors, MITF/TFE family members can bind the TCATGTG M-box sequence (Napolitano and Ballabio, 2016). Within family members, the domains outside BHLH-LZ are quite different. TFEB has a glutamine-rich domain (Gln) and a proline-rich domain (Pro) (Oppezzo and Rosselli, 2021). MITF and TFEC has a serine-rich domain (Ser), while TFE3 has a proline- and arginine-rich domain (Pro + Arg) (Oppezzo and Rosselli, 2021). In invertebrates, orthologs of MITF/TFE family also exist, including helix-loop-helix transcription factor 30 (HLH-30) in Caenorhabditis elegans (Rehli et al., 1999a) and MITF in Drosophila melanogaster (Hallsson et al., 2004). They both have basic domains and HLH-zip domains similar to those MITF/TFE family members, and can bind DNA in a similar manner (Bouché et al., 2016).

Results by real-time RT-PCR showed that the MiTF/TFE family was regulated by multiple levels, indicating that these transcription factors might be involved in multiple intracellular processes among disparate tissues (Kuiper et al., 2004). It has been documented that TFEB, TFE3, and MITF share the same regulatory effects in autophagy (Sardiello et al., 2009; Martina et al., 2014; Bouché et al., 2016; Ozturk et al., 2019), which further influence host immune response (Irazoqui, 2020). Indeed, the distinct features of each transcription factor are noteworthy. For example, TFEB is capable of regulating mitochondrial biosynthesis and exerting quality control of mitochondria (Settembre et al., 2013; Nezich et al., 2015; Theeuwes et al., 2020). Meanwhile, MITF is reportedly involved in the development of retinal pigment epithelial and melanocytes (Levy et al., 2006; Ma et al., 2019), and TFE3 is needed for the Golgi stress response (Taniguchi and Yoshida, 2017) and expression of insulin signaling genes (Nakagawa et al., 2006; Iwasaki et al., 2012). However, TFEC appears to be structurally different from the other transcription factors due to lack of acidic domain, thereby functioning as transcriptional inhibition rather than activation (Zhao et al., 1993). TFEC is specifically expressed in bone marrow-derived cells (Rehli et al., 1999b), and is involved in regulating hematopoietic function of hematopoietic stem cells (Mahony et al., 2016).

\section{THE REGULATION OF TFEB ACTIVITY}

A growing body of evidence shows that the transcriptional activity of TFEB is mainly determined by subcellular localization, which largely depends on its phosphorylating status (Puertollano et al., 2018). Under the condition of nutritional sufficiency, TFEB accumulates in the cytoplasm and remains in the form of inactivation by phosphorylation, while it is dephosphorylated and activated in response to starvation, and subsequently transfers to the nucleus for initiating genes transcription. The convert between phosphorylation and dephosphorylation of TFEB and its cytoplasmic-nucleus shuttling are regulated by multiple pathways (Figure 1).

\section{The Phosphorylation of Cytoplasmic TFEB}

The mammalian target of rapamycin complex 1 (mTORC1) is the most widely studied signaling for mediating phosphorylation 


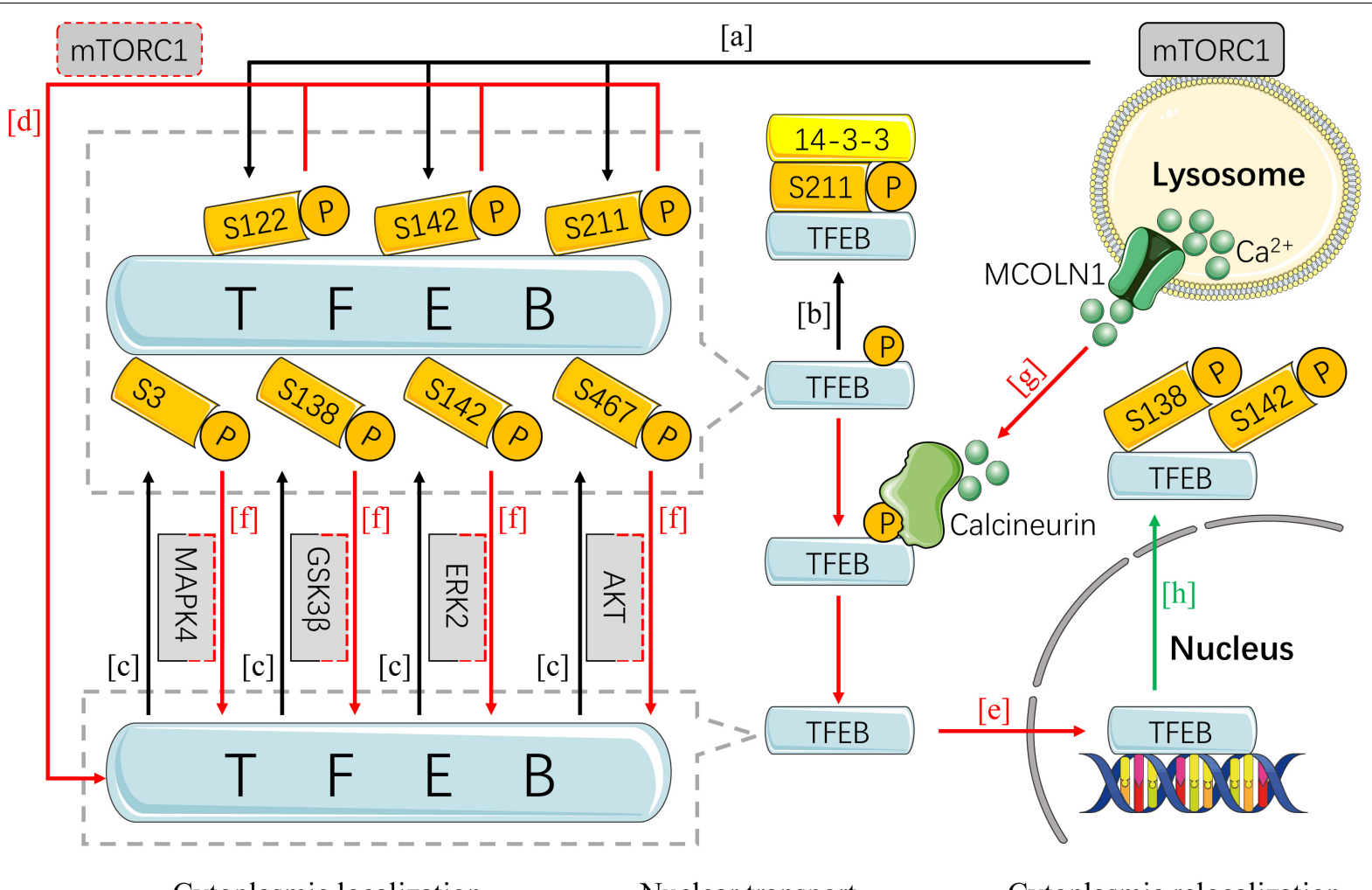

Cytoplasmic localization

Nuclear transport

Cytoplasmic relocalization

FIGURE 1 | The regulation of TFEB activity by phosphorylation. In the condition of nutritional sufficiency, TFEB is isolated outside the nucleus in an inactive state by phosphorylation and accumulates in the cytoplasm. TFEB can be phosphorylated by mTORC1 on S122, S142, and S211 (A), by AKT on S467, and by GSK3 $\beta$ on S138, by ERK2 on S142, and by MAPK4 on S3 (C). The phosphorylated TFEB on S211 is recognized and bound by YWHA/14-3-3 protein in atypical patterns (B). When encountering with starvation or stress, the withdraw of any phosphorylation site is sufficient to induce TFEB activation and nuclear transport (D,F). Calcineurin is stimulated by the $\mathrm{Ca}^{2+}$ released from the lysosome in MCOLN1-dependent way and is primarily responsible for the dephosphorylation of TFEB (G).

Dephosphorylated TFEB transfers to nucleus and further initiates the transcription of target genes (E). Refeeding after nutrition deprivation, TFEB initiates cytoplasmic relocalization in a short time by hierarchical phosphorylation on S142 and S138 (H). (AKT, protein kinase B; ERK2, extracellular regulated protein kinase 2; GSK3 $\beta$, glycogen synthase kinase 3ß; MAPK4, mitogen-activated protein kinase 4; mTORC1, mammalian target of rapamycin complex 1; TFEB, transcription factor EB).

of TFEB, which is essential for cellular metabolism, growth, and survival via integrating environmental input into downstream pathways (Ardestani et al., 2018). Upon sufficient nutritional supplementation, mTORC1 is recruited to the surface of lysosomes and activated by amino acid and growth factors (Long et al., 2005; Sancak et al., 2010), thereby promoting phosphorylation of TFEB. Up to now, three serine sites have been found responsible for the phosphorylation of TFEB by mTORC1, including S142 (Settembre et al., 2012), S211 (Martina et al., 2012; Roczniak-Ferguson et al., 2012; Settembre et al., 2012), and recently discovered S122 (Vega-Rubin-de-Celis et al., 2017; Figure 1A). It has been demonstrated that the inactive form of TFEB is maintained mainly by phosphorylation of S142 and S211 (Martina et al., 2012; Roczniak-Ferguson et al., 2012), while the phosphorylation of TFEB on S211 is recognized and bound by YWHA/14-3-3 protein in atypical patterns, which inhibits its nuclear translocation (Martina et al., 2012; Xu et al., 2019; Figure 1B).

In addition to mTORC1, TFEB is noticed to be phosphorylated and inhibited by other factors, including protein kinase $\mathrm{B}$ (AKT) on phosphorylation of S467 (Palmieri et al., 2017b), glycogen synthase kinase $3 \beta$ (GSK3 $\beta$ ) on S138 (Li Y. et al., 2016), extracellular regulated protein kinase 2 (ERK2) on S142, and mitogen-activated protein kinase 4 (MAPK4) on S3 (Hsu et al., 2018; Figure 1C). Nevertheless, their relationship with cytoplasmic retention of TFEB remains largely unknown.

\section{Nuclear Transfer of TFEB by Dephosphorylation}

The covert between phosphorylation and dephosphorylation status is the determinant for TFEB activation and nuclear transfer. In the setting of starvation, mTORC1 dissociates from the lysosome due to inactivation, which further induces the dephosphorylation of TFEB (Figure 1D). The latter subsequently enters the nucleus for augmenting transcriptional activity of targeted genes followed with dissociation from YWHA/143-3 (Martina et al., 2012; Roczniak-Ferguson et al., 2012; Figure 1E). Similarly, withdraw of AKT, GSK3 $\beta$ by the activation of protein kinase C (PKC), ERK2, and MAP4K3 on TFEB phosphorylation is sufficient to initiate TFEB activation and nuclear translocation (Settembre et al., 2011; Li Y. et al., 2016; 
Palmieri et al., 2017b; Hsu et al., 2018; Figure 1F). In addition, activation of AMP-activated protein kinase (AMPK) promotes the activation of TFEB through inhibiting mTORC1 pathway (van Veelen et al., 2011; Brady et al., 2018a). AMPK can also upregulate dephosphorylation and nuclear localization of TFEB independently of mTORC1 (Collodet et al., 2019). And pharmacological activation of AMPK or inducing loss of FLCN, the negative regulator of AMPK, will inhibit this progress (El-Houjeiri et al., 2019).

Calcineurin is thought to be responsible for the dephosphorylation of TFEB, which can be activated by lysosomal $\mathrm{Ca}^{2+}$ (Medina et al., 2015; Figure 1G). Endoplasmic reticulum stress and reactive oxygen species (ROS) can promote the effects of calcineurin on TFEB directly or indirectly through mucolipin 1 (MCOLN1), a lysosomal $\mathrm{Ca}^{2+}$ channel (Martina et al., 2016; Zhang et al., 2016). However, a recent study has shown that proteasome inhibition leads to a significant increase in TFEB dephosphorylation level and nuclear translocation ( $\mathrm{Li}$ et al., 2019), hinting critical involvement of ubiquitin proteasome pathway on TFEB activation, which needs further elucidation on precise mechanism and discrepancy across disparate studies.

\section{Nuclear Export of TFEB by Re-phosphorylation}

It has been clarified that the intracellular localization of TFEB is the determinant of its activation, namely shuttle between cytoplasm and nucleus. Likewise, delayed or disturbed exportation of nuclear TFEB might pose threat to the intracellular homeostasis. Two recent studies have shown that refeeding after nutrition deprivation results in cytoplasmic relocalization of TFEB in a short time through inducing hierarchical phosphorylation of S142 and S138, suggesting that re-phosphorylation of nuclear TFEB is the major mechanism for its cytoplasmic relocalization (Li et al., 2018a; Napolitano et al., 2018; Figure 1H). Study conducted by Napolitano et al. (2018) also identified a highly evolutionary conserved sequence in the $\mathrm{N}$-terminal of TFEB, named nuclear export signal (NES) due to its indispensable role in the cytoplasmic relocalization of TFEB, which was largely impaired by NES mutation. Intriguingly, both the S142 and S138 are located near NES (Napolitano et al., 2018), implying the possible role for NES on the re-phosphorylation of TFEB at both sites. Notably, it has been indicated that inhibition of XPO1, a nuclear export protein, can facilitate the nuclear localization of TFEB without affecting mTOR activity (Silvestrini et al., 2018). However, study that deals with nuclear export of TFEB is still rare, let alone its specific mechanism.

\section{Other Post-transcriptional Modifications of TFEB}

In addition to phosphorylation, other post-transcriptional modifications are also involved in the regulation of TFEB. For instance, the acetylation of TFEB at K274 and K279 affects its transcriptional activity by disrupting dimerization and inhibiting binding to promoters (Wang Y. et al., 2020), which can be reversed by deacetylation (Bao et al., 2016). The SUMOylation of TFEB at a lysine site will lead to decreased transcriptional activity (Miller et al., 2005), while the ubiquitination and further degradation of phosphorylated TFEB will facilitate TFEB activation via ubiquitin-proteasome pathway (Sha et al., 2017).

\section{The Self-Regulation of TFEB}

Interestingly, targeted molecules of TFEB are in turn involved in regulating TFEB activity, implicating the self-regulation of TFEB (Figure 2). For example, MCOLN1 is responsible for TFEB dephosphorylation by inducing the release of $\mathrm{Ca}^{2+}$ from lysosomes (Medina et al., 2015; Figure 2Da). Cathepsin B, a subtype of hydrolase located within the lysosomal lumen, downregulates the activation of TFEB by degrading MCOLN1 (Man and Kanneganti, 2016; Figure 2Da). V-ATPase, the $\mathrm{H}^{+}$pump on lysosomal membrane, can activate TFEB nuclear translocation after disrupting its catalytic subunit A (Kim D. et al., 2017; Figure 2Da). TFEB-mediated endocytosis enhances the assembling and activation of mTORC1, which is closely relatedto TFEB phosphorylation on lysosomal membranes through prompting the formation of endosomes (Nnah et al., 2019; Figure 2Db). TFEB is indeed essential for the autophagic process, while its activation is also commonly evident during lysosomal damage or autophagy disruption (Settembre et al., 2011; Roczniak-Ferguson et al., 2012; Figure 2Dc). Moreover, TFEB directly activates peroxisome proliferator-activated receptor $\gamma$ coactivator $1 \alpha$ (PGC-1 $\alpha)$, and it can be activated in a PGC1-dependent manner (Tsunemi et al., 2012; Kim Y.S. et al., 2017; Erlich et al., 2018; Figure 2Dd). Notably, it was revealed that knockout of either TFEB or PGC-1 resulted in decreased expression of the other gene (Scott et al., 2014). In addition, TFEB was found to be associated with the activation of mitophagy by regulating PTEN-induced putative kinase 1 (PINK1)/Parkin signaling pathway, and its expression was also influenced by PINK1/Parkin signaling (Ivankovic et al., 2016; Figure 2De).

\section{THE FUNCTION OF TFEB}

Activated TFEB can transfer to nucleus and initiates transcription of various genes. TFEB contributes to the maintenance of cellular structure and function via promoting the biosynthesis of multiple organelles and autophagic activities. TFEB also plays a role in host defense machinery by regulating host immune response.

\section{The Effects of TFEB on Biosynthesis and Function of Multiple Organelles}

Lysosome serves as the digestive center of eukaryotic cells, and it is programmed to recycle and degrade biomacromolecule by means of phagocytosis, endocytosis, and autophagy, thereby providing raw materials for cellular anabolism. Meanwhile, it can act as a signaling hub accounting for the main regulatory mechanism of nutrition sensing (Lawrence and Zoncu, 2019). Activation of TFEB is capable of upregulating the expression of multiple lysosomal proteins by identifying and combining the CLEAR elements, which is the promoter region of multiple lysosomal genes (Sardiello et al., 2009). Studies have been demonstrated that TFEB not only regulates lysosomal 

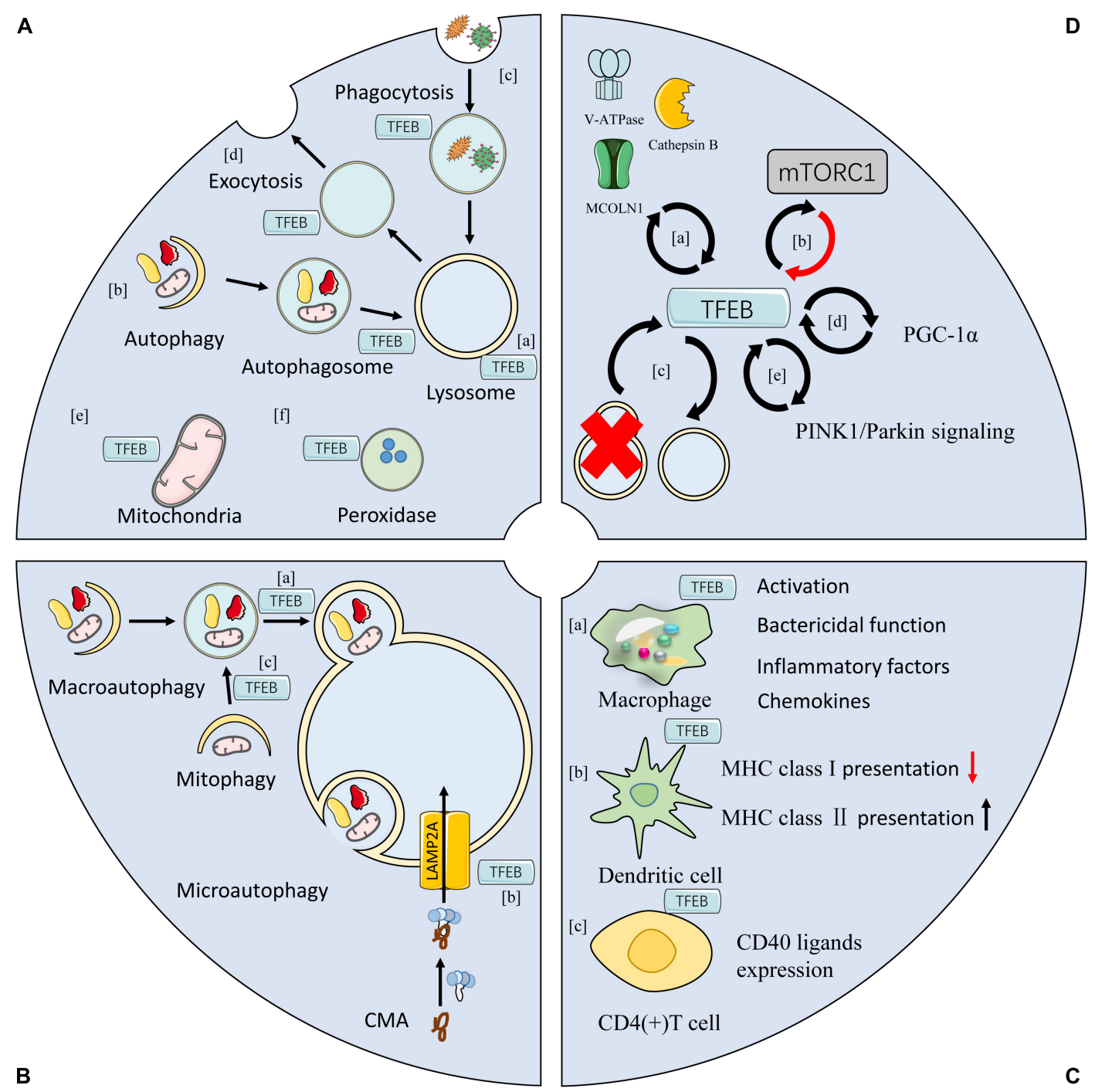

FIGURE 2 | Functions of TFEB. (A) TFEB is involved in the biogenesis of lysosomes (a), mitochondrial (e), peroxidases (f), and autophagosomes (b). (B) TFEB participates in multiple types of autophagy, including macroautophagy, microautophagy, and chaperone mediated autophagym (CMA) by regulating the function of lysosomes. It is also involved in autophagy at various steps, such as the fusion of autophagosome with lysosome in macroautophagy (a), the localization and trafficking of LAMP2A in CMA (b), and the priming the clearance of damaged mitochondrial in mitophagy (c). (C) TFEB regulates immune responses in both innate and adaptive immune systems. TFEB is involved in activation, bactericidal capacity, and production of inflammatory mediators in macrophages (a). In dendritic cells, TFEB inhibits the antigen presentation of MHC class I but enhances the expression of MHC class II (b). In T cells, TFEB is related to antibody response in reaction to the activation of CD40 at cellular surface (c). (D) The modulation of TFEB activity. Lysosomal proteins, such as V-ATPase, cathepsin B, and MCOLN1 that are induced by TFEB, can regulate TFEB activity in return (a). mTORC1 is critically involved in the TFEB phosphorylation, which can be activated by TFEB (b). TFEB is a major regulator of ALP, and both lysosomal damage and autophagy disruption result in TFEB activation (c). TFEB directly activate PGC-1 $\alpha$, and it can be activated in a PGC-1 $\alpha$-dependent manner (d). TFEB induces mitophagy by activating PINK1/Parkin pathway, and its activity is also enhanced by stimulating PINK1/Parkin (e). (mTORC1, mammalian target of rapamycin complex 1; CMA, chaperone mediated autophagy; PGC-1 $\alpha$, peroxisome proliferator-activated receptor $\gamma$ coactivator $1 \alpha$; TFEB, transcription factor EB; MHC, major histocompatibility complex).

biosynthesis (Figure 2Aa), but enhances the expression of multiple genes governing lysosomal related processes, including autophagy, phagocytosis, and exocytosis (Palmieri et al., 2011; Figures $\mathbf{2 A b}-\mathbf{d}$ ). For example, activation of TFEB was identified to augment the bactericidal performance of macrophages via promoting its phagocytic activity (Gray et al., 2016). TFEB was found to facilitate lysosomal fusion with the plasma membrane and exocytosis by triggering activation of MCOLN1 in $\mathrm{Ca}^{2+}$ dependent mechanism (Medina et al., 2011). Furthermore, TFEB was reportedly capable of regulating the location of lysosomes by increasing TMEM55B expression (Willett et al., 2017).

Mitochondria is the intracellular "energy factory" which plays an important role in host immunity, inflammation, and metabolism (Spinelli and Haigis, 2018; Breda et al., 2019; Vringer and Tait, 2019). TFEB is involved in mitochondrial biosynthesis by inducing the expression of PGC-1 $\alpha$, the major 
factor for regulating mitochondrial biosynthesis (Ma et al., 2015; Erlich et al., 2018; Figure 2Ae). TFEB mediates the increased expression of multiple mitochondrial proteins in PGC- $1 \alpha$ independent manners. For example, mitochondrial transcription factor A (TFAM) is a key factor in directly regulating mtDNA transcription and can be activated by nuclear respiratory factor (NRF)1 and NRF2 (Farge and Falkenberg, 2019). In skeletal muscle of PGC-1 $\alpha$ knockout mice, overexpression of TFEB was noted to upregulate the expression of NRF1, NRF2, and TFAM, and mitochondrial volume as well as density simultaneously (Mansueto et al., 2017). In addition, TFEB is essential for mitochondrial stability in mitochondrial dynamics through reducing mitochondrial division (Santin et al., 2016). Functionally, activation of TFEB optimizes mitochondrial substrate utilization, which is favorable for respiratory function and accelerates the production of ATP (Mansueto et al., 2017; Zhang et al., 2019), while it may lead to the accumulation of dysfunctional mitochondria when inhibiting TFEB activity (Zhang et al., 2019).

Recently, TFEB was found to be capable of controlling the biogenesis of peroxidases at the transcriptional level (Figure 2Af), as shown by significantly reduced levels of peroxidases due to inhibition of TFEB (Eun et al., 2018). Furthermore, inhibition of mTORC1 was reported to alleviate the abnormal biogenesis of peroxidases (Eun et al., 2018), which could be partly attributed to the counteract of TFEB inhibition. Additionally, TFEB promoted the biosynthesis of autophagosomes and its subsequent fusion with lysosomes (Settembre et al., 2011).

\section{Regulatory Impacts on Autophagy}

Autophagy refers to a tight regulated process that transports a variety of cellular components to lysosomes for degradation, which involves the renewal of multiple organelles and the removal of misfolded proteins and even invaded intracellular pathogens. Strikingly, TFEB is essential for the completion of all types of autophagy, including macroautophagy, microautophagy, and CMA, by regulating the function and number of lysosomes.

In fact, TFEB has been confirmed involving in multiple steps of autophagic process. For example, macroautophagy, deemed as a highly conserved catabolic process, is clarified to be comprised of various steps, including formation of autophagosomes and further fusion with lysosomes, as well as cargos degradation ( $\mathrm{Yu}$ et al., 2018). TFEB is required for the biosynthesis of autophagosomes and its fusion with lysosomes (Settembre et al., 2011; Figure 2Ba). CMA is completed by transporting and degrading target proteins in a molecular chaperone dependent manner (Kaushik and Cuervo, 2018). Lysosome-associated membrane protein 2A (LAMP2A) is the firstly identified lysosomal component for the completion of CMA. Of note, activation of TFEB enhances CMA by facilitating the trafficking and localization of LAMP2A (Zhang et al., 2017; Figure 2Bb). Similarly, TFEB participates in identifying cargos during the initiation of selective autophagy. Taking mitophagy as an example, one of its priming mechanism is PINK1/Parkin signaling pathway, which is reportedly TFEB-dependent (Kim et al., 2018; Figure 2Bc). Activation of TFEB can promote the recruitment of autophagosomes to the surface of mitochondria (Tan et al., 2019b).

\section{Immune and Inflammatory Responses}

TFEB is involved in host response by regulating both innate and adaptive immunity (Brady et al., 2018b; Irazoqui, 2020). It has been documented that TFEB participates in the process of macrophage activation by following mechanisms (Fang L. et al., 2017), including promoting bactericidal function (Gray et al., 2016; Liu X. et al., 2019) and facilitating the production of various proinflammatory mediators (Visvikis et al., 2014; Pastore et al., 2016; Figure 2Ca). TFEB is demonstrated to play a role in innate immune response by modulating xenophagy, a form of selective autophagy targeting invaded microorganisms (Wang and Li, 2020). For example, persistent activation of nuclear TFEB resulted in the scavenging activity of functional lysosomes, which further suppressed the replication of Salmonellas via targeting Salmonella-containing vacuoles (Ammanathan et al., 2019). Moreover, TFEB was essential for the function of dendritic cells (DCs) when encountering with different exogenous antigens (Figure $\mathbf{2} \mathbf{C b}$ ). It has been identified that activation of TFEB inhibits the antigen-presentation capacity of major histocompatibility complex (MHC) class I, but upregulates the expression of MHC class II (Samie and Cresswell, 2015). Likely, TFEB is critically involved in T-cell-dependent antibiotic activity by cooperating with TFE3 in response to the activation of CD40 on the surface of $\mathrm{T}$ cells (Huan et al., 2006; Figure 2Cc). In addition, TFEB functions as a proinflammatory mediator by promoting the lysosomal degradation of anti-inflammatory cytokines (He et al., 2011).

As a critical component of immune and inflammatory responses is regulated by TFEB. TFEB can be activated by $\mathrm{Ca}^{2+}$ signaling to induce lysosomal endocytosis and further promote IL-1 $\beta$ secretion in human monocytes (Tseng et al., 2017). It also collaborates with TFE3 to induce gene transcription of proinflammatory cytokines in activated macrophages and microglia (Pastore et al., 2016). While in TFEB and TFE3 deficient cells, the secretion of key inflammatory mediators, including CSF2, IL1 $\beta$, IL2, and IL27, was significantly suppressed (Pastore et al., 2016), supporting the role of TFEB in regulating inflammatory response. Moreover, TFEB can induce the expression of peroxisome proliferator activator receptor $\alpha$ (PPAR- $\alpha$ ) and PGC- $1 \alpha$ to promote mitochondrial biogenesis and thus indirectly reduce ROS production and inflammation (Kim Y.S. et al., 2017).

In addition, TFEB has been found to play a role in controlling metabolism including lipid, energy, amino acid and glucose (Pastore et al., 2017). TFEB takes a part in the metabolism of lipid by regulating lipid transport and lipophagy (Zechner et al., 2012), while overexpression of TFEB perturbs expressions of numerous genes covering fatty acid-binding, transport and oxidation (Settembre et al., 2013). What's more, transcriptome analysis in murine cardiomyocytes with TFEB depletion revealed that TFEB regulates a gene network involved in lipid and carbohydrate metabolism independently of macroautophagy (Trivedi et al., 2020). TFEB also responds to the disturbance in energy balance. The increased energy demand during exercise can provoke 
TFEB expression and activation to improve energy metabolism through mitochondrial biogenesis and mitophagy (Erlich et al., 2018). The cellular response to amino acid signal is primarily via RAGs, heterodimers formed by the combination of RagA/B and RagC/D (Zhu et al., 2020). It has been demonstrated that defective mTOR-TFEB signaling is involved in the progression of $\operatorname{RagC}^{S 75 Y}$ mutated cardiomyopathy, which can only be corrected by overexpression of TFEB but not inhibition of mTOR (Kim et al., 2021). Therefore, TFEB may play a role in amino acid metabolism by regulating RagC and need further study to verify. As for glucose metabolism, TFEB can control the expressions of glucose transporters, glycolytic enzymes and glucose homeostasis pathways (Mansueto et al., 2017). And glucose uptake can be increased by TFEB in ways of activating AKT and upregulating insulin receptor substrate 1 (IRS1) and IRS2 (Sun et al., 2021).

\section{THE ROLE OF TFEB IN HUMAN DISEASES}

Since TFEB is essential for maintaining the stability of cellular structure and function, the dysregulation of TFEB activity appears to be associated with the occurrence and development of various human diseases, such as tumors, neurodegenerative diseases, and inflammatory diseases, implying the modulation of TFEB a potential therapeutic target (Table 1).

\section{Tumors}

Hyperactive catabolism is the underlying mechanism for the uncontrolled growth and metastasis of the tumor. It has been reported that TFEB may contribute to the development and progression of tumor by upregulating autophagy and endocytosis to meet the metabolic requirement in pancreatic and prostate cancer cells (Perera et al., 2015; Blessing et al., 2017). TFEB can inhibit the apoptosis of breast cancer cells by activating DNA repair (Slade et al., 2020). And the tumor progression of breast cancer can be inhibited by macrophagespecific TFEB overexpression through enhancing the functional status of immune cells within the tumor microenvironment, as inhibition of TFEB in macrophages obviously leads to the growth of breast cancer (Fang L. et al., 2017). TFEB also mediates tumor metastasis. For example, in a mouse model of lung cancer with liver metastasis, TFEB augmented the metastasis by increasing autophagy through upregulating cathepsins secretion and lysosome synthesis (Kundu et al., 2018). In fact, the TFEB shows divergent impacts on the treatments of various kinds of cancers. With regard to the melanoma and multiple myeloma, for example, elevated expression of TFEB presented anticancer effects via triggering autophagic cell death (Cea et al., 2013; Silvente-Poirot et al., 2018). In breast cancer, however, the partial functional loss of TFEB by the interaction of signal transducer and activator of transcription 3 (STAT3) enhances lysosomal-mediated cell death, thus serving as an anticancer action ( $\mathrm{Li}$ et al., 2018b). In glioblastoma, inhibition of TFEB resulted in the death of tumor cells due to its incapacity of withstanding growing metabolic pressure (Sung et al., 2019). TFEB is found to be involved in chemoresistance in cancer. For
TABLE 1 | TFEB serves as a potential therapeutic target for human diseases.

\begin{tabular}{|c|c|c|c|}
\hline Diseases & & $\begin{array}{l}\text { TFEB as a disease } \\
\text { inductor }\end{array}$ & $\begin{array}{l}\text { TFEB as a } \\
\text { treatment target }\end{array}$ \\
\hline \multirow[t]{4}{*}{ Tumors } & $\begin{array}{l}\text { Pancreatic } \\
\text { cancer }\end{array}$ & $\begin{array}{l}\text { Increased TFEB } \\
\text { promotes autophagy } \\
\text { to meet the metabolic } \\
\text { needs for growth and } \\
\text { metastasis of tumor } \\
\text { cells }\end{array}$ & $\begin{array}{l}\text { Inhibition of TFEB } \\
\text { enhances the } \\
\text { sensitivity to } \\
\text { chemotherapy }\end{array}$ \\
\hline & Lung cancer & $\begin{array}{l}\text { Upregulated TFEB } \\
\text { enhances the release } \\
\text { of cathepsins to } \\
\text { facilitate metastasis; } \\
\text { Increased TFEB } \\
\text { develops resistance } \\
\text { to chemotherapy }\end{array}$ & $\begin{array}{l}\text { Down-regulation of } \\
\text { TFEB augments the } \\
\text { sensitivity to } \\
\text { chemotherapy }\end{array}$ \\
\hline & Breast cancer & $\begin{array}{l}\text { Activated DNA repair } \\
\text { inhibits apoptosis of } \\
\text { tumor cells }\end{array}$ & $\begin{array}{l}\text { Inhibition of TFEB in } \\
\text { breast cancer cells } \\
\text { promotes tumor cell } \\
\text { death; upregulated } \\
\text { TFEB in } \\
\text { macrophages } \\
\text { enhances the } \\
\text { inhibition of tumor } \\
\text { progression }\end{array}$ \\
\hline & TFEB RCC & $\begin{array}{l}\text { Translocation or } \\
\text { rearrangement of } \\
\text { TFEB gene leads to } \\
\text { nuclear TFEB } \\
\text { overexpression }\end{array}$ & - \\
\hline \multirow[t]{3}{*}{$\begin{array}{l}\text { Inflammatory } \\
\text { diseases }\end{array}$} & Atherosclerosis & - & $\begin{array}{l}\text { Antioxidation in } \\
\text { endothelial cells; the } \\
\text { enhancement of } \\
\text { lysosomal function in } \\
\text { macrophages; } \\
\text { lipophagy promotion } \\
\text { in vascular smooth } \\
\text { muscle cells. }\end{array}$ \\
\hline & Sepsis & - & $\begin{array}{l}\text { Autophagy } \\
\text { restoration by } \\
\text { upregulation of TFEB }\end{array}$ \\
\hline & Osteoarthritis & $\begin{array}{l}\text { Inhibited expression } \\
\text { and nuclear import of } \\
\text { TFEB }\end{array}$ & $\begin{array}{l}\text { Autophagy promotion } \\
\text { by TFEB } \\
\text { overexpression }\end{array}$ \\
\hline \multirow[t]{3}{*}{$\begin{array}{l}\text { Neurodege } \\
\text { nerative } \\
\text { diseases }\end{array}$} & $\begin{array}{l}\text { Parkinson } \\
\text { disease }\end{array}$ & $\begin{array}{l}\text { Cytoplasmic retention } \\
\text { of TFEB leads to } \\
\text { decreased lysosomal } \\
\text { function and } \\
\text { excessive } \alpha \text {-synuclein } \\
\text { production }\end{array}$ & $\begin{array}{l}\text { Activation of TFEB } \\
\text { reverses lysosomal } \\
\text { dysfunction and } \\
\text { reduces oxidative } \\
\text { stress }\end{array}$ \\
\hline & $\begin{array}{l}\text { Alzheimer } \\
\text { disease }\end{array}$ & - & $\begin{array}{l}\text { Elevated TFEB } \\
\text { expression or } \\
\text { deacetylation of TFEB } \\
\text { improves the } \\
\text { degradation of tau }\end{array}$ \\
\hline & $\begin{array}{l}\text { Huntington } \\
\text { disease }\end{array}$ & $\begin{array}{l}\text { Reduced TFEB level } \\
\text { and target genes } \\
\text { expression }\end{array}$ & $\begin{array}{l}\text { Activation or } \\
\text { overexpression of } \\
\text { TFEB increases the } \\
\text { clearance of HTT }\end{array}$ \\
\hline
\end{tabular}

instance, TFEB reduced the sensitivity of LoVo and HeLa cells to doxorubicin by inducing activation of autophagy (Fang L. M. et al., 2017), and it reduced the apoptosis of breast cancer cells 
in response to doxorubicin by repairing DNA damage (Slade et al., 2020). In addition, increased TFEB expression was noticed in both glioblastoma and lung cancer developing resistance to chemotherapy (Karagounis et al., 2016; Sung et al., 2019). Given that, inhibition of TFEB by gene silencing or drugs can markedly enhance the sensitivity of tumor cells to chemotherapy among glioblastoma, lung cancer, pancreatic cancer, and ovarian cancer through either impairing autophagy flux or inducing mitochondrial apoptosis (Karagounis et al., 2016; He et al., 2018; Mitrakas et al., 2018; Song et al., 2019). Therefore, exploring anticancer strategies underlying regulation of TFEB should be based on distinct tumor types.

Recently, TFEB was noticed to be closely associated with the development of $\mathrm{t}(6 ; 11)$ renal cell carcinoma (RCC), an extremely rare subtype that occurred in adolescents and accounted for $0.02 \%$ of all kidney cancers (Inamura, 2017). Since $t(6 ; 11)$ translocation fuses the TFEB gene on chromosome 6 with the metastasis associated lung adenocarcinoma transcript 1 (MALAT1) gene on chromosome 11, the rearrangement of the fused gene consequently retains the entire TFEB coding sequence and further induces the promoter activity of MALAT1, thereby resulting in the overexpression of nuclear TFEB (Zhan et al., 2018; Caliò et al., 2019). Another phenotype of TFEB-related RCC named TFEB-amplified RCC is independent of TFEB rearrangement, which has been identified sharing feature of nuclear TFEB overexpression similar to t $(6 ; 11)$ RCC (Caliò et al., 2019), but occurring in older patients with a more aggressive clinical course (Argani et al., 2016). Currently, the mechanism underlying RCC development by TFEB overexpression remains unclear. The mouse model of renal carcinoma due to specific renal TFEB overexpression was established, and it was found that highly activated Wnt pathway played an important role in TFEB-induced RCC. Antagonizing Wnt pathway could restrain the proliferation of cancer cells and relieved or even completely reversed the pathological phenotype of RCC (Calcagnì et al., 2016).

\section{Inflammatory Diseases}

Dysregulation of TFEB leads to the imbalance of inflammatory response, which is one of the major causes for inflammatory diseases. TFEB has been shown to play a protective role in endothelial cells via migrating oxidative stress and upregulating the expression of multiple antioxidant genes, thereby slowing down the progression of atherosclerosis ( $\mathrm{Lu}$ et al., 2017). As the atherosclerosis progressed, macrophages are recruited into the growing plaques for the clearance of deposited lipids and apoptotic cells. While TFEB can enhance the lysosomal function in macrophages and prompt the phenotypic shift of macrophages to anti-inflammation subtype, thereby reducing the burden on atherosclerotic plaques (Sergin et al., 2017; Evans et al., 2018; You et al., 2020). While Stearyl coenzyme A desaturase 1 (SCD1) can promote TFEB-mediated lipophagy to interfere with foam cell formation and modulation of the SCD1/TFEB machinery may offer novel therapeutic approaches for atherosclerosis (Pi et al., 2019).

As we known, autophagy is considered to be an important self-protective mechanism for cell survival, with great potential in maintaining immune homeostasis and alleviating multiorgan failure under septic challenge (Ren et al., 2017). Since TFEB serves as a regulatory element in autophagic initiation, it reveals great potential in attenuating lethal septic response. In the mouse model of sepsis-induced cardiac dysfunction, TFEB-mediated autophagy showed obvious beneficial for migrating myocardial injury (Li F. et al., 2016).

In osteoarthritis (OA), the expression and nuclear transportation of TFEB presented with marked inhibition in both human and mouse chondrocytes (Zheng et al., 2018). It was reported that in the mouse OA model, TFEB overexpression by lentiviral transfection improved cartilage degradation and down-regulated chondrocyte apoptosis and senescence by enhancing autophagy, which might be a promising therapeutic candidate for OA treatment (Zheng et al., 2018). Accumulating evidences have indicated TFEB plays emerging role in the onset and development of other inflammatory diseases. For example, decreased TFEB expression through the phosphorylation by ERK increases inflammation and mitochondrial damage in the lung tissue and alveolar epithelial cells, contributing to the occurrence of pneumonia (Liu W. et al., 2019). Elevated phosphorylated level and cytoplasmic retention of TFEB lead to blocked lysosomal autophagy pathway and enhanced pancreatic proteasome activity, thus leading to pancreatitis (Wang et al., 2019). TFEB deficiency can also lead to the downregulation of lipoprotein ApoA1 which is linked to colitis susceptibility (Murano et al., 2017). Given that, TFEB maybe a new target for intervention and treatment of various inflammatory diseases.

\section{Neurodegenerative Diseases}

One of the evident pathological features of neurodegenerative diseases is the accumulation of abnormal proteins, such as $\alpha$-synuclein in Parkinson disease (PD), amyloid $\beta(\mathrm{A} \beta)$ and tau in Alzheimer disease (AD), huntingtin (HTT) in Huntington disease, polyglutamine-expanded androgen receptor (polyQ-AR) in X-linked spinal and bulbar muscular atrophy (SBMA), and mutant superoxide dismutase 1 (SOD1) in amyotrophic lateral sclerosis (ALS). These abnormal aggregates largely attribute to the impairment of autophagy-lysosomal function, which is reportedly closely associated with the activity of TFEB.

In a model of $\alpha$-synuclein toxicity, excessive $\alpha$-synuclein production in the nigral dopamine neurons was closely related to the decline of lysosomal function caused by cytoplasmic retention of TFEB (Decressac et al., 2013). While delayed activation of TFEB function through inhibition of mTORC1 blocked $\alpha$-synuclein induced neurodegeneration and further disease progression (Decressac et al., 2013). In the mouse model of $\mathrm{AD}$ with tau spreading, exogenous TFEB expression was extensively noted in astrocytes, which was capable of capturing and degrading tau, thus markedly alleviating tau-associated pathological alterations (Martini-Stoica et al., 2018). In a mouse model of $\mathrm{AD}$, the improvement of pathological changes could be augmented by exogenous supplementation of TFEB (Polito et al., 2014), which was achieved by inducing autophagy and reducing tau via promoting lysosomal exocytosis (Xu et al., 2020). The A $\beta$ was degraded by enhanced lysosomal function via deacetylation of TFEB in microglia (Bao et al., 2016). In the mouse model 
of Huntington disease, the level of TFEB and the expression of its targeted gene were found with significant reduction. TFEB activation by restoration of PGC- $1 \alpha$ is sufficient to reduce HTT aggregation and neurotoxicity (Tsunemi et al., 2012). Besides, trehalose was capable of activating TFEB by downregulating $\mathrm{AKT}$ activity, thereby increasing the clearance of protein aggregations and reducing neuropathologic phenotypes (Palmieri et al., 2017a; Rusmini et al., 2019), while silencing TFEB significantly disturbed the pro-degradation activity of trehalose (Rusmini et al., 2019). SBMA is characterized by proximal muscle weakness due to the abnormal accumulation of polyQ$\mathrm{AR}$, which causes degeneration of the lower motor neurons in the spinal cord and brain stem (Cortes and La Spada, 2019). The activation of TFEB is noted in normal AR, but reveals interference by polyQ-AR, further impairing autophagy and promoting the pathogenesis of SBMA (Cortes et al., 2014). While TFEB overexpression by the upregulation of nuclear factor-YA markedly promoted the clearance of pathogenic AR protein in motor neurons and muscles of SBMA mouse model, thus mitigating the behavioral and pathological impairments (Tohnai et al., 2014). ALS is a motor neuron disorder characterized by abnormal accumulation of mutant SOD1 (Cortes and La Spada, 2019). Dysregulated TFEB localization was found in brain samples of ALS patients (Wang et al., 2016), while overexpression of TFEB by plasmid induced autophagy to clear accumulated SOD1, thus maintaining cell survival and proliferation (Chen et al., 2015). Therefore, TFEB is critically involved in the pathophysiological process of various neurodegenerative diseases as its key role in regulating lysosomal function, and maintaining TFEB activity can be considered as an effective way to accelerate the clearance of abnormally accumulated proteins.

\section{CONCLUSION AND PERSPECTIVE}

With the deepening understanding of TFEB, its regulatory pathway, specific function, and relationship with the onset of multiple diseases have been uncovered, making TFEB an indispensable transcription factor for cellular homeostasis. Upstream regulators of TFEB have been well studied. Inhibitors including mTORC1, AKT, GSK3 $\beta$, ERK2, and MAPK4 act mainly through the phosphorylation of TFEB. Activation of TFEB takes effects either by dephosphorization, such as calcineurin, or by inhibiting TFEB cascades, such as AMPK and PKC for down-regulation of $\mathrm{mTORC1}$ and GSK3 $\beta$, respectively. Kruppel-like factor 15 (KLF15) is another discovered TFEB activator that augments TFEB protein content and nuclear translocation in the cardiomyocyte (Trivedi et al., 2020). While the exact mechanism of KLF15 regulating TFEB still merits further investigation. Among the downstream TFEB effectors, the CLEAR elements, PPAR- $\alpha$, and PGC- $1 \alpha$ are the most studied. Regarding the regulation of TFEB activity, cytoplasmic retention and nuclear transfer have been extensively reported by quantities of studies, while the precise mechanism underlying nuclear export remains further elucidated. Although phosphorylation has been accepted as the main mechanism for TFEB activation, other modifications, like acetylation, need further clarification.
Given the important role of TFEB in the pathogenesis of many human diseases, the current understanding of TFEBrelated human diseases mainly focuses on its association with lysosome and autophagy, which has been identified involving in catabolic hyperactivity in tumors, imbalanced host responses in inflammatory diseases, and accumulation of abnormal aggregates in neurodegenerative diseases.

Therapies targeting TFEB might exert promising impacts on disease remission. With regard to the great heterogeneity among various malignancies, disparate strategies can be adopted under specific circumstances. For tumor cells of melanoma and multiple myeloma, the expression of TFEB is upregulated to present with anticancer effects via triggering autophagic cell death. Nevertheless, down-regulation of TFEB is needed to increase chemotherapy sensitivity for tumor cells with drug resistance. In inflammatory diseases, the pathological phenotypes can be relieved via upregulation of TFEB which is able to migrate oxidative stress in response to inflammatory imbalance and facilitate the clearance of inflammatory substances through autophagy. As for neurodegenerative diseases, the accumulation of pathogenic proteins due to lysosomal dysfunction can be alleviated by the upregulation of TFEB activity.

Currently, TFEB-related drugs have been developed. A synthesized curcumin derivative termed $\mathrm{C} 1$ enhances nuclear translocation of TFEB through specifically binding to the N-terminal of TFEB (Song et al., 2016), and it has been proved to ameliorate beta-amyloid precursor protein and tau pathology in Alzheimer's disease models (Song et al., 2020). Other drugs, including resveratrol, progestin R5020, and 3,4-dimethoxychalcone are also reported to augment TFEB translocation into nucleus and enhance the function of TFEB (Chen et al., 2019; Tan et al., 2019a; Zhou et al., 2019). In addition, there are few reports with regard to TFEB inhibitors, e.g., Mn induces decreased nuclear localization of TFEB and results in autophagy dysfunction in astrocytes of mouse striatum (Zhang et al., 2020). Taken together, an understanding of the biological significance and pathophysiological roles of TFEB is favorable for further exploring novel yet efficient therapeutic measures for human diseases.

\section{AUTHOR CONTRIBUTIONS}

S-YZ and R-QY: literature investigation and data curation. S-YZ, R-QY, X-HD, CR, and Y-MY: writing-original draft, figure preparation, writing-review and editing. Y-XL and P-YZ: writingreview and editing. All authors contributed to the article and approved the submitted version.

\section{FUNDING}

This work was supported by grants from the National Natural Science Foundation (81730057 and 81801935), the National Key Research and Development Program of China (2017YFC1103302), and the China Postdoctoral Science Foundation (2019M664003). 


\section{REFERENCES}

Aksan, I., and Goding, C. R. (1998). Targeting the microphthalmia basic helix-loophelix-leucine zipper transcription factor to a subset of E-box elements in vitro and in vivo. Mol. Cell Biol. 18, 6930-6938. doi: 10.1128/mcb.18.12.6930

Ammanathan, V., Mishra, P., Chavalmane, A. K., Muthusamy, S., Jadhav, V., Siddamadappa, C., et al. (2019). Restriction of intracellular Salmonella replication by restoring TFEB-mediated xenophagy. Autophagy 2019, 1-14.

Ardestani, A., Lupse, B., Kido, Y., Leibowitz, G., and Maedler, K. (2018). mTORC1 Signaling: A Double-Edged Sword in Diabetic $\beta$ Cells. Cell Metab. 27, 314-331. doi: 10.1016/j.cmet.2017.11.004

Argani, P., Reuter, V. E., Zhang, L., Sung, Y. S., Ning, Y., Epstein, J. I., et al. (2016). TFEB-amplified Renal Cell Carcinomas: An Aggressive Molecular Subset Demonstrating Variable Melanocytic Marker Expression and Morphologic Heterogeneity. Am. J. Surg. Pathol. 40, 1484-1495. doi: 10.1097/ pas. 0000000000000720

Bao, J., Zheng, L., Zhang, Q., Li, X., Zhang, X., Li, Z., et al. (2016). Deacetylation of TFEB promotes fibrillar $\mathrm{A} \beta$ degradation by upregulating lysosomal biogenesis in microglia. Protein Cell 7, 417-433. doi: 10.1007/s13238-0160269-2

Blessing, A. M., Rajapakshe, K., Reddy Bollu, L., Shi, Y., White, M. A., Pham, A. H., et al. (2017). Transcriptional regulation of core autophagy and lysosomal genes by the androgen receptor promotes prostate cancer progression. Autophagy 13, 506-521. doi: 10.1080/15548627.2016.1268300

Bouché, V., Espinosa, A. P., Leone, L., Sardiello, M., Ballabio, A., and Botas, J. (2016). Drosophila Mitf regulates the V-ATPase and the lysosomalautophagic pathway. Autophagy 12, 484-498. doi: 10.1080/15548627.2015.11 34081

Brady, O. A., Jeong, E., Martina, J. A., Pirooznia, M., Tunc, I., and Puertollano, R. (2018a). The transcription factors TFE 3 and TFEB amplify p 53 dependent transcriptional programs in response to DNA damage. Elife 2018:7.

Brady, O. A., Martina, J. A., and Puertollano, R. (2018b). Emerging roles for TFEB in the immune response and inflammation. Autophagy 14, 181-189. doi: 10.1080/15548627.2017.1313943

Breda, C. N. S., Davanzo, G. G., Basso, P. J., Saraiva Câmara, N. O., and MoraesVieira, P. M. M. (2019). Mitochondria as central hub of the immune system. Redox Biol. 26:101255. doi: 10.1016/j.redox.2019.101255

Calcagnì, A., Kors, L., Verschuren, E., De Cegli, R., Zampelli, N., Nusco, E., et al. (2016). Modelling TFE renal cell carcinoma in mice reveals a critical role of WNT signaling. eLife 2016:5.

Caliò, A., Segala, D., Munari, E., Brunelli, M., and Martignoni, G. (2019). MiT Family Translocation Renal Cell Carcinoma: from the Early Descriptions to the Current Knowledge. Cancers 2019:11.

Cea, M., Cagnetta, A., Patrone, F., Nencioni, A., Gobbi, M., and Anderson, K. C. (2013). Intracellular $\mathrm{NAD}(+)$ depletion induces autophagic death in multiple myeloma cells. Autophagy 9, 410-412. doi: 10.4161/auto.22866

Chen, G., Xie, W., Nah, J., Sauvat, A., Liu, P., Pietrocola, F., et al. (2019). 3,4Dimethoxychalcone induces autophagy through activation of the transcription factors TFE3 and TFEB. EMBO Mol. Med. 11:e10469.

Chen, Y., Liu, H., Guan, Y., Wang, Q., Zhou, F., Jie, L., et al. (2015). The altered autophagy mediated by TFEB in animal and cell models of amyotrophic lateral sclerosis. Am. J. Transl. Res. 7, 1574-1587.

Collodet, C., Foretz, M., Deak, M., Bultot, L., Metairon, S., Viollet, B., et al. (2019). AMPK promotes induction of the tumor suppressor FLCN through activation of TFEB independently of mTOR. Faseb J. 33, 12374-12391. doi: 10.1096/fj. $201900841 \mathrm{r}$

Cortes, C. J., and La Spada, A. R. (2019). TFEB dysregulation as a driver of autophagy dysfunction in neurodegenerative disease: Molecular mechanisms, cellular processes, and emerging therapeutic opportunities. Neurobiol. Dis. 122, 83-93. doi: 10.1016/j.nbd.2018.05.012

Cortes, C. J., Miranda, H. C., Frankowski, H., Batlevi, Y., Young, J. E., Le, A., et al. (2014). Polyglutamine-expanded androgen receptor interferes with TFEB to elicit autophagy defects in SBMA. Nat. Neurosci. 17, 1180-1189. doi: 10.1038/ nn. 3787

Decressac, M., Mattsson, B., Weikop, P., Lundblad, M., Jakobsson, J., and Björklund, A. (2013). TFEB-mediated autophagy rescues midbrain dopamine neurons from $\alpha$-synuclein toxicity. Proc. Natl. Acad. Sci. U S A 110, E1817E1826.
El-Houjeiri, L., Possik, E., Vijayaraghavan, T., Paquette, M., Martina, J. A., Kazan, J. M., et al. (2019). The Transcription Factors TFEB and TFE3 Link the FLCNAMPK Signaling Axis to Innate Immune Response and Pathogen Resistance. Cell Rep. 26, 3613.e-3628.e.

Erlich, A. T., Brownlee, D. M., Beyfuss, K., and Hood, D. A. (2018). Exercise induces TFEB expression and activity in skeletal muscle in a PGC-1 $\alpha-$ dependent manner. Am. J. Physiol. Cell Physiol. 314, C62-C72.

Eun, S. Y., Lee, J. N., Nam, I. K., Liu, Z. Q., So, H. S., Choe, S. K., et al. (2018). PEX5 regulates autophagy via the mTORC1-TFEB axis during starvation. Exp. Mol. Med. 50:4.

Evans, T. D., Jeong, S. J., Zhang, X., Sergin, I., and Razani, B. (2018). TFEB and trehalose drive the macrophage autophagy-lysosome system to protect against atherosclerosis. Autophagy 14, 724-726. doi: 10.1080/15548627.2018.1434373

Fang, L., Hodge, J., Saaoud, F., Wang, J., Iwanowycz, S., Wang, Y., et al. (2017). Transcriptional factor EB regulates macrophage polarization in the tumor microenvironment. Oncoimmunology 6:e1312042. doi: 10.1080/2162402x.2017. 1312042

Fang, L. M., Li, B., Guan, J. J., Xu, H. D., Shen, G. H., Gao, Q. G., et al. (2017). Transcription factor EB is involved in autophagy-mediated chemoresistance to doxorubicin in human cancer cells. Acta Pharmacol. Sin. 38, 1305-1316. doi: 10.1038 /aps.2017.25

Farge, G., and Falkenberg, M. (2019). Organization of DNA in Mammalian Mitochondria. Int. J. Mol. Sci. 2019:20.

Gray, M. A., Choy, C. H., Dayam, R. M., Ospina-Escobar, E., Somerville, A., Xiao, X., et al. (2016). Phagocytosis Enhances Lysosomal and Bactericidal Properties by Activating the Transcription Factor TFEB. Curr. Biol. 26, 1955-1964. doi: 10.1016/j.cub.2016.05.070

Hallsson, J. H., Haflidadóttir, B. S., Stivers, C., Odenwald, W., Arnheiter, H., Pignoni, F., et al. (2004). The basic helix-loop-helix leucine zipper transcription factor Mitf is conserved in Drosophila and functions in eye development. Genetics 167, 233-241. doi: 10.1534/genetics.167.1.233

He, R., Shi, X., Zhou, M., Zhao, Y., Pan, S., Zhao, C., et al. (2018). Alantolactone induces apoptosis and improves chemosensitivity of pancreatic cancer cells by impairment of autophagy-lysosome pathway via targeting TFEB. Toxicol. Appl. Pharmacol. 356, 159-171. doi: 10.1016/j.taap.2018.08.003

He, Y., Xu, Y., Zhang, C., Gao, X., Dykema, K. J., Martin, K. R., et al. (2011). Identification of a lysosomal pathway that modulates glucocorticoid signaling and the inflammatory response. Sci. Signal 4:ra44. doi: 10.1126/scisignal. 2001450

Hemesath, T. J., Steingrímsson, E., McGill, G., Hansen, M. J., Vaught, J., Hodgkinson, C. A., et al. (1994). microphthalmia, a critical factor in melanocyte development, defines a discrete transcription factor family. Genes Dev. 8, 2770-2780. doi: 10.1101/gad.8.22.2770

Hsu, C. L., Lee, E. X., Gordon, K. L., Paz, E. A., Shen, W. C., Ohnishi, K., et al. (2018). MAP4K3 mediates amino acid-dependent regulation of autophagy via phosphorylation of TFEB. Nat. Commun. 9:942.

Huan, C., Kelly, M. L., Steele, R., Shapira, I., Gottesman, S. R., and Roman, C. A. (2006). Transcription factors TFE 3 and TFEB are critical for CD40 ligand expression and thymus-dependent humoral immunity. Nat. Immunol. 7, 1082-1091. doi: 10.1038/ni1378

Inamura, K. (2017). Translocation Renal Cell Carcinoma: An Update on Clinicopathological and Molecular Features. Cancers 2017:9.

Irazoqui, J. E. (2020). Key Roles of MiT Transcription Factors in Innate Immunity and Inflammation. Trends Immunol. 41, 157-171. doi: 10.1016/j.it.2019.12.003

Ivankovic, D., Chau, K. Y., Schapira, A. H., and Gegg, M. E. (2016). Mitochondrial and lysosomal biogenesis are activated following PINK1/parkin-mediated mitophagy. J. Neurochem. 136, 388-402. doi: 10.1111/jnc.13412

Iwasaki, H., Naka, A., Iida, K. T., Nakagawa, Y., Matsuzaka, T., Ishii, K. A., et al. (2012). TFE3 regulates muscle metabolic gene expression, increases glycogen stores, and enhances insulin sensitivity in mice. Am. J. Physiol. Endocrinol. Metab. 302, E896-E902.

Karagounis, I. V., Kalamida, D., Mitrakas, A., Pouliliou, S., Liousia, M. V., Giatromanolaki, A., et al. (2016). Repression of the autophagic response sensitises lung cancer cells to radiation and chemotherapy. Br. J. Cancer 115, 312-321. doi: 10.1038/bjc.2016.202

Kaushik, S., and Cuervo, A. M. (2018). The coming of age of chaperone-mediated autophagy. Nat. Rev. Mol. Cell Biol. 19, 365-381. doi: 10.1038/s41580-0180001-6 
Kim, D., Hwang, H. Y., Kim, J. Y., Lee, J. Y., Yoo, J. S., Marko-Varga, G., et al. (2017). FK506, an Immunosuppressive Drug, Induces Autophagy by Binding to the V-ATPase Catalytic Subunit A in Neuronal Cells. J. Proteome Res. 16, 55-64. doi: 10.1021/acs.jproteome.6b00638

Kim, H. J., Joe, Y., Rah, S. Y., Kim, S. K., Park, S. U., Park, J., et al. (2018). Carbon monoxide-induced TFEB nuclear translocation enhances mitophagy/mitochondrial biogenesis in hepatocytes and ameliorates inflammatory liver injury. Cell Death Dis. 9:1060.

Kim, M., Lu, L., Dvornikov, A. V., Ma, X., Ding, Y., Zhu, P., et al. (2021). TFEB Overexpression, Not mTOR Inhibition, Ameliorates RagC(S75Y) Cardiomyopathy. Int. J. Mol. Sci. 2021:22.

Kim, Y. S., Lee, H. M., Kim, J. K., Yang, C. S., Kim, T. S., Jung, M., et al. (2017). PPAR- $\alpha$ Activation Mediates Innate Host Defense through Induction of TFEB and Lipid Catabolism. J. Immunol. 198, 3283-3295. doi: 10.4049/jimmunol. 1601920

Kuiper, R. P., Schepens, M., Thijssen, J., Schoenmakers, E. F., and van Kessel, A. G. (2004). Regulation of the MiTF/TFE bHLH-LZ transcription factors through restricted spatial expression and alternative splicing of functional domains. Nucleic Acids Res. 32, 2315-2322. doi: 10.1093/nar/gkh571

Kundu, S. T., Grzeskowiak, C. L., Fradette, J. J., Gibson, L. A., Rodriguez, L. B., Creighton, C. J., et al. (2018). TMEM106B drives lung cancer metastasis by inducing TFEB-dependent lysosome synthesis and secretion of cathepsins. Nat. Comm. 9:2731.

Lawrence, R. E., and Zoncu, R. (2019). The lysosome as a cellular centre for signalling, metabolism and quality control. Nat. Cell Biol. 21, 133-142. doi: 10.1038/s41556-018-0244-7

Levy, C., Khaled, M., and Fisher, D. E. (2006). MITF: master regulator of melanocyte development and melanoma oncogene. Trends Mol. Med. 12, 406-414. doi: 10.1016/j.molmed.2006.07.008

Li, C., Wang, X., Li, X., Qiu, K., Jiao, F., Liu, Y., et al. (2019). Proteasome Inhibition Activates Autophagy-Lysosome Pathway Associated With TFEB Dephosphorylation and Nuclear Translocation. Front. Cell Dev. Biol. 7:170.

Li, F., Lang, F., Zhang, H., Xu, L., Wang, Y., and Hao, E. (2016). Role of TFEB Mediated Autophagy, Oxidative Stress, Inflammation, and Cell Death in Endotoxin Induced Myocardial Toxicity of Young and Aged Mice. Oxid. Med. Cell Longev. 2016:5380319.

Li, L., Friedrichsen, H. J., Andrews, S., Picaud, S., Volpon, L., Ngeow, K., et al. (2018a). A TFEB nuclear export signal integrates amino acid supply and glucose availability. Nat. Commun. 9:2685.

Li, L., Sun, B., Gao, Y., Niu, H., Yuan, H., and Lou, H. (2018b). STAT3 contributes to lysosomal-mediated cell death in a novel derivative of riccardin D-treated breast cancer cells in association with TFEB. Biochem. Pharmacol. 150, 267-279. doi: 10.1016/j.bcp.2018.02.026

Li, Y., Xu, M., Ding, X., Yan, C., Song, Z., Chen, L., et al. (2016). Protein kinase C controls lysosome biogenesis independently of mTORC1. Nat. Cell Biol. 18, 1065-1077. doi: 10.1038/ncb3407

Liu, W., Li, C. C., Lu, X., Bo, L. Y., and Jin, F. G. (2019). Overexpression of transcription factor $\mathrm{EB}$ regulates mitochondrial autophagy to protect lipopolysaccharide-induced acute lung injury. Chin. Med. J. 132, 1298-1304. doi: $10.1097 / \mathrm{cm} 9.0000000000000243$

Liu, X., Zhang, N., Liu, Y., Liu, L., Zeng, Q., Yin, M., et al. (2019). MPB, a novel berberine derivative, enhances lysosomal and bactericidal properties via TGF$\beta$-activated kinase 1-dependent activation of the transcription factor EB. Faseb J. 33, 1468-1481. doi: 10.1096/fj.201801198r

Long, X., Lin, Y., Ortiz-Vega, S., Yonezawa, K., and Avruch, J. (2005). Rheb binds and regulates the mTOR kinase. Curr. Biol. 15, 702-713. doi: 10.1016/j.cub. 2005.02.053

Lu, H., Fan, Y., Qiao, C., Liang, W., Hu, W., Zhu, T., et al. (2017). TFEB inhibits endothelial cell inflammation and reduces atherosclerosis. Sci. Signal 2017:10,

Ma, X., Li, H., Chen, Y., Yang, J., Chen, H., Arnheiter, H., et al. (2019). The transcription factor MITF in RPE function and dysfunction. Prog. Retin. Eye Res. 73:100766. doi: 10.1016/j.preteyeres.2019.06.002

Ma, X., Liu, H., Murphy, J. T., Foyil, S. R., Godar, R. J., Abuirqeba, H., et al. (2015). Regulation of the transcription factor EB-PGC1 $\alpha$ axis by beclin- 1 controls mitochondrial quality and cardiomyocyte death under stress. Mol. Cell Biol. 35 , 956-976.

Mahony, C. B., Fish, R. J., Pasche, C., and Bertrand, J. Y. (2016). tfec controls the hematopoietic stem cell vascular niche during zebrafish embryogenesis. Blood 128, 1336-1345. doi: 10.1182/blood-2016-04-7 10137

Man, S. M., and Kanneganti, T. D. (2016). Regulation of lysosomal dynamics and autophagy by CTSB/cathepsin B. Autophagy 12, 2504-2505. doi: 10.1080/ 15548627.2016.1239679

Mansueto, G., Armani, A., Viscomi, C., D’Orsi, L., De Cegli, R., Polishchuk, E. V., et al. (2017). Transcription Factor EB Controls Metabolic Flexibility during Exercise. Cell Metab. 25, 182-196. doi: 10.1016/j.cmet.2016.11.003

Martina, J. A., Chen, Y., Gucek, M., and Puertollano, R. (2012). MTORC1 functions as a transcriptional regulator of autophagy by preventing nuclear transport of TFEB. Autophagy 8, 903-914. doi: 10.4161/auto.19653

Martina, J. A., Diab, H. I., Brady, O. A., and Puertollano, R. (2016). TFEB and TFE3 are novel components of the integrated stress response. Embo J. 35, 479-495. doi: $10.15252 / \mathrm{embj} .201593428$

Martina, J. A., Diab, H. I., Lishu, L., Jeong, A. L., Patange, S., Raben, N., et al. (2014). The nutrient-responsive transcription factor TFE3 promotes autophagy, lysosomal biogenesis, and clearance of cellular debris. Sci. Signal 7:ra9. doi: $10.1126 /$ scisignal.2004754

Martini-Stoica, H., Cole, A. L., Swartzlander, D. B., Chen, F., Wan, Y. W., Bajaj, L., et al. (2018). TFEB enhances astroglial uptake of extracellular tau species and reduces tau spreading. J. Exp. Med. 215, 2355-2377. doi: 10.1084/jem.20172158

Martini-Stoica, H., Xu, Y., Ballabio, A., and Zheng, H. (2016). The AutophagyLysosomal Pathway in Neurodegeneration: A TFEB Perspective. Trends Neurosci. 39, 221-234. doi: 10.1016/j.tins.2016.02.002

Medina, D. L., Di Paola, S., Peluso, I., Armani, A., De Stefani, D., Venditti, R., et al. (2015). Lysosomal calcium signalling regulates autophagy through calcineurin and TFEB. Nat. Cell Biol. 17, 288-299. doi: 10.1038/ncb3114

Medina, D. L., Fraldi, A., Bouche, V., Annunziata, F., Mansueto, G., Spampanato, C., et al. (2011). Transcriptional activation of lysosomal exocytosis promotes cellular clearance. Dev. Cell 21, 421-430. doi: 10.1016/j.devcel.2011.07.016

Miller, A. J., Levy, C., Davis, I. J., Razin, E., and Fisher, D. E. (2005). Sumoylation of MITF and its related family members TFE3 and TFEB. J. Biol. Chem. 280, 146-155. doi: 10.1074/jbc.m411757200

Mitrakas, A. G., Kalamida, D., Giatromanolaki, A., Pouliliou, S., Tsolou, A., Kyranas, R., et al. (2018). Autophagic flux response and glioblastoma sensitivity to radiation. Cancer Biol. Med. 15, 260-274. doi: 10.20892/j.issn.2095-3941. 2017.0173

Muhle-Goll, C., Gibson, T., Schuck, P., Schubert, D., Nalis, D., Nilges, M., et al. (1994). The dimerization stability of the HLH-LZ transcription protein family is modulated by the leucine zippers: a CD and NMR study of TFEB and c-Myc. Biochemistry 33, 11296-11306. doi: 10.1021/bi0020 $3 \mathrm{a} 027$

Murano, T., Najibi, M., Paulus, G. L. C., Adiliaghdam, F., Valencia-Guerrero, A., Selig, M., et al. (2017). Transcription factor TFEB cell-autonomously modulates susceptibility to intestinal epithelial cell injury in vivo. Sci. Rep. 7:13938.

Nakagawa, Y., Shimano, H., Yoshikawa, T., Ide, T., Tamura, M., Furusawa, M., et al. (2006). TFE3 transcriptionally activates hepatic IRS-2, participates in insulin signaling and ameliorates diabetes. Nat. Med. 12, 107-113. doi: 10.1038/nm1334

Napolitano, G., and Ballabio, A. (2016). TFEB at a glance. J. Cell Sci. 129, 24752481.

Napolitano, G., Esposito, A., Choi, H., Matarese, M., Benedetti, V., Di Malta, C., et al. (2018). mTOR-dependent phosphorylation controls TFEB nuclear export. Nat. Commun. 9:3312.

Nezich, C. L., Wang, C., Fogel, A. I., and Youle, R. J. (2015). MiT/TFE transcription factors are activated during mitophagy downstream of Parkin and Atg5. J. Cell Biol. 210, 435-450. doi: 10.1083/jcb.201501002

Nnah, I. C., Wang, B., Saqcena, C., Weber, G. F., Bonder, E. M., Bagley, D., et al. (2019). TFEB-driven endocytosis coordinates MTORC1 signaling and autophagy. Autophagy 15, 151-164. doi: 10.1080/15548627.2018.1511504

Oppezzo, A., and Rosselli, F. (2021). The underestimated role of the microphthalmia-associated transcription factor (MiTF) in normal and pathological haematopoiesis. Cell Biosci. 11:18.

Ozturk, D. G., Kocak, M., Akcay, A., Kinoglu, K., Kara, E., Buyuk, Y., et al. (2019). MITF-MIR211 axis is a novel autophagy amplifier system during cellular stress. Autophagy 15, 375-390. doi: 10.1080/15548627.2018.1531197

Palmieri, M., Impey, S., Kang, H., di Ronza, A., Pelz, C., Sardiello, M., et al. (2011). Characterization of the CLEAR network reveals an integrated control of cellular clearance pathways. Hum. Mol. Genet. 20, 3852-3866. doi: 10.1093/hmg/ddr306 
Palmieri, M., Pal, R., Nelvagal, H. R., Lotfi, P., Stinnett, G. R., Seymour, M. L., et al. (2017b). mTORC1-independent TFEB activation via Akt inhibition promotes cellular clearance in neurodegenerative storage diseases. Nat. Commun. 8:14338.

Palmieri, M., Pal, R., Nelvagal, H. R., Lotfi, P., Stinnett, G. R., Seymour, M. L., et al. (2017a). Corrigendum: mTORC1-independent TFEB activation via Akt inhibition promotes cellular clearance in neurodegenerative storage diseases. Nat. Commun. 8:15793.

Pastore, N., Brady, O. A., Diab, H. I., Martina, J. A., Sun, L., Huynh, T., et al. (2016). TFEB and TFE3 cooperate in the regulation of the innate immune response in activated macrophages. Autophagy 12, 1240-1258. doi: 10.1080/15548627.2016. 1179405

Pastore, N., Vainshtein, A., Klisch, T. J., Armani, A., Huynh, T., Herz, N. J., et al. (2017). TFE3 regulates whole-body energy metabolism in cooperation with TFEB. EMBO Mol. Med. 9, 605-621. doi: 10.15252/emmm.2016 07204

Perera, R. M., Stoykova, S., Nicolay, B. N., Ross, K. N., Fitamant, J., Boukhali, M., et al. (2015). Transcriptional control of autophagy-lysosome function drives pancreatic cancer metabolism. Nature 524, 361-365. doi: 10.1038/nature14587

Pi, H., Wang, Z., Liu, M., Deng, P., Yu, Z., Zhou, Z., et al. (2019). SCD1 activation impedes foam cell formation by inducing lipophagy in oxLDL-treated human vascular smooth muscle cells. J. Cell Mol. Med. 23, 5259-5269. doi: 10.1111/ jcmm.14401

Pogenberg, V., Ogmundsdóttir, M. H., Bergsteinsdóttir, K., Schepsky, A., Phung, B., Deineko, V., et al. (2012). Restricted leucine zipper dimerization and specificity of DNA recognition of the melanocyte master regulator MITF. Genes Dev. 26, 2647-2658. doi: 10.1101/gad.198192.112

Polito, V. A., Li, H., Martini-Stoica, H., Wang, B., Yang, L., Xu, Y., et al. (2014). Selective clearance of aberrant tau proteins and rescue of neurotoxicity by transcription factor EB. EMBO Mol. Med. 6, 1142-1160. doi: 10.15252/emmm. 201303671

Puertollano, R., Ferguson, S. M., Brugarolas, J., and Ballabio, A. (2018). The complex relationship between TFEB transcription factor phosphorylation and subcellular localization. Embo J. 2018:37.

Rehli, M., Den Elzen, N., Cassady, A. I., Ostrowski, M. C., and Hume, D. A. (1999a). Cloning and characterization of the murine genes for bHLH-ZIP transcription factors TFEC and TFEB reveal a common gene organization for all MiT subfamily members. Genomics 56, 111-120. doi: 10.1006/geno.1998.5588

Rehli, M., Lichanska, A., Cassady, A. I., Ostrowski, M. C., and Hume, D. A. (1999b). TFEC is a macrophage-restricted member of the microphthalmiaTFE subfamily of basic helix-loop-helix leucine zipper transcription factors J. Immunol. 162, 1559-1565.

Ren, C., Zhang, H., Wu, T. T., and Yao, Y. M. (2017). Autophagy: A Potential Therapeutic Target for Reversing Sepsis-Induced Immunosuppression. Front. Immunol. 8:1832.

Roczniak-Ferguson, A., Petit, C. S., Froehlich, F., Qian, S., Ky, J., Angarola, B., et al. (2012). The transcription factor TFEB links mTORC1 signaling to transcriptional control of lysosome homeostasis. Sci. Signal 5:ra42. doi: 10.1126/ scisignal.2002790

Rusmini, P., Cortese, K., Crippa, V., Cristofani, R., Cicardi, M. E., Ferrari, V., et al. (2019). Trehalose induces autophagy via lysosomal-mediated TFEB activation in models of motoneuron degeneration. Autophagy 15, 631-651. doi: 10.1080/ 15548627.2018.1535292

Samie, M., and Cresswell, P. (2015). The transcription factor TFEB acts as a molecular switch that regulates exogenous antigen-presentation pathways. Nat. Immunol. 16, 729-736. doi: 10.1038/ni.3196

Sancak, Y., Bar-Peled, L., Zoncu, R., Markhard, A. L., Nada, S., and Sabatini, D. M. (2010). Ragulator-Rag complex targets mTORC1 to the lysosomal surface and is necessary for its activation by amino acids. Cell 141, 290-303. doi: 10.1016/j. cell.2010.02.024

Santin, Y., Sicard, P., Vigneron, F., Guilbeau-Frugier, C., Dutaur, M., Lairez, O., et al. (2016). Oxidative Stress by Monoamine Oxidase-A Impairs Transcription Factor EB Activation and Autophagosome Clearance, Leading to Cardiomyocyte Necrosis and Heart Failure. Antioxid. Redox Signal 25, 10-27. doi: 10.1089/ars.2015.6522

Sardiello, M., Palmieri, M., di Ronza, A., Medina, D. L., Valenza, M., Gennarino, V. A., et al. (2009). A gene network regulating lysosomal biogenesis and function. Science 325, 473-477. doi: 10.1126/science.1174447
Scott, I., Webster, B. R., Chan, C. K., Okonkwo, J. U., Han, K., and Sack, M. N. (2014). GCN5-like protein 1 (GCN5L1) controls mitochondrial content through coordinated regulation of mitochondrial biogenesis and mitophagy. J. Biol. Chem. 289, 2864-2872. doi: 10.1074/jbc.m113.521641

Sergin, I., Evans, T. D., Zhang, X., Bhattacharya, S., Stokes, C. J., Song, E., et al. (2017). Exploiting macrophage autophagy-lysosomal biogenesis as a therapy for atherosclerosis. Nat. Commun. 8:15750.

Settembre, C., De Cegli, R., Mansueto, G., Saha, P. K., Vetrini, F., Visvikis, O., et al. (2013). TFEB controls cellular lipid metabolism through a starvation-induced autoregulatory loop. Nat. Cell Biol. 15, 647-658. doi: 10.1038/ncb2718

Settembre, C., Di Malta, C., Polito, V. A., Garcia Arencibia, M., Vetrini, F., Erdin, S., et al. (2011). TFEB links autophagy to lysosomal biogenesis. Science 332, 1429-1433.

Settembre, C., Zoncu, R., Medina, D. L., Vetrini, F., Erdin, S., Erdin, S., et al. (2012). A lysosome-to-nucleus signalling mechanism senses and regulates the lysosome via mTOR and TFEB. Embo J. 31, 1095-1108. doi: 10.1038/emboj.2012.32

Sha, Y., Rao, L., Settembre, C., Ballabio, A., and Eissa, N. T. (2017). STUB1 regulates TFEB-induced autophagy-lysosome pathway. Embo J. 36, 2544-2552. doi: 10.15252/embj.201796699

Silvente-Poirot, S., Segala, G., Poirot, M. C., and Poirot, M. (2018). Liganddependent transcriptional induction of lethal autophagy: a new perspective for cancer treatment. Autophagy 14, 555-557. doi: 10.1080/15548627.2018.1425059

Silvestrini, M. J., Johnson, J. R., Kumar, A. V., Thakurta, T. G., Blais, K., Neill, Z. A., et al. (2018). Nuclear Export Inhibition Enhances HLH-30/TFEB Activity, Autophagy, and Lifespan. Cell Rep. 23, 1915-1921. doi: 10.1016/j.celrep.2018. 04.063

Slade, L., Biswas, D., Ihionu, F., El Hiani, Y., Kienesberger, P. C., and Pulinilkunnil, T. (2020). A lysosome independent role for TFEB in activating DNA repair and inhibiting apoptosis in breast cancer cells. Biochem. J. 477, 137-160. doi: 10.1042/bcj20190596

Slade, L., and Pulinilkunnil, T. (2017). The MiTF/TFE Family of Transcription Factors: Master Regulators of Organelle Signaling, Metabolism, and Stress Adaptation. Mol. Cancer Res. 15, 1637-1643. doi: 10.1158/1541-7786.mcr-170320

Song, J. X., Malampati, S., Zeng, Y., Durairajan, S. S. K., Yang, C. B., Tong, B. C., et al. (2020). A small molecule transcription factor EB activator ameliorates beta-amyloid precursor protein and Tau pathology in Alzheimer's disease models. Aging Cell 19:e13069.

Song, J. X., Sun, Y. R., Peluso, I., Zeng, Y., Yu, X., Lu, J. H., et al. (2016). A novel curcumin analog binds to and activates TFEB in vitro and in vivo independent of MTOR inhibition. Autophagy 12, 1372-1389. doi: 10.1080/15548627.2016. 1179404

Song, X., Liu, L., Chang, M., Geng, X., Wang, X., Wang, W., et al. (2019). NEO212 induces mitochondrial apoptosis and impairs autophagy flux in ovarian cancer. J. Exp. Clin. Cancer Res. 38:239.

Spinelli, J. B., and Haigis, M. C. (2018). The multifaceted contributions of mitochondria to cellular metabolism. Nat. Cell Biol. 20, 745-754. doi: 10.1038/ s41556-018-0124-1

Sun, J., Lu, H., Liang, W., Zhao, G., Ren, L., Hu, D., et al. (2021). Endothelial TFEB (Transcription Factor EB) Improves Glucose Tolerance via Upregulation of IRS (Insulin Receptor Substrate) 1 and IRS2. Arterioscler. Thromb. Vasc. Biol. 41, 783-795. doi: 10.1161/atvbaha.120.315310

Sung, G. J., Kim, S. H., Kwak, S., Park, S. H., Song, J. H., Jung, J. H., et al. (2019). Inhibition of TFEB oligomerization by co-treatment of melatonin with vorinostat promotes the therapeutic sensitivity in glioblastoma and glioma stem cells. J. Pineal Res. 66:e12556. doi: 10.1111/jpi.12556

Tan, S., Bajalovic, N., Wong, E. S. P., and Lin, V. C. L. (2019a). Ligand-activated progesterone receptor $\mathrm{B}$ activates transcription factor $\mathrm{EB}$ to promote autophagy in human breast cancer cells. Exp. Cell Res. 382:111433. doi: 10.1016/j.yexcr. 2019.05.014

Tan, S., Yu, C. Y., Sim, Z. W., Low, Z. S., Lee, B., See, F., et al. (2019b). Pomegranate activates TFEB to promote autophagy-lysosomal fitness and mitophagy. Sci. Rep. 9:727.

Taniguchi, M., and Yoshida, H. (2017). TFE3, HSP47, and CREB3 Pathways of the Mammalian Golgi Stress Response. Cell Struct. Funct. 42, 27-36. doi: 10.1247/ csf. 16023

Theeuwes, W. F., Gosker, H. R., Schols, A., Langen, R. C. J., and Remels, A. H. V. (2020). Regulation of PGC- $1 \alpha$ expression by a GSK-3 $\beta$-TFEB signaling axis 
in skeletal muscle. Biochim. Biophys. Acta Mol. Cell Res. 1867:118610. doi: 10.1016/j.bbamcr.2019.118610

Tohnai, G., Adachi, H., Katsuno, M., Doi, H., Matsumoto, S., Kondo, N., et al. (2014). Paeoniflorin eliminates a mutant AR via NF-YA-dependent proteolysis in spinal and bulbar muscular atrophy. Hum. Mol. Genet. 23, 3552-3565. doi: $10.1093 / \mathrm{hmg} / \mathrm{ddu} 066$

Trivedi, P. C., Bartlett, J. J., Mercer, A., Slade, L., Surette, M., Ballabio, A., et al. (2020). Loss of function of transcription factor EB remodels lipid metabolism and cell death pathways in the cardiomyocyte. Biochim. Biophys. Acta Mol. Basis Dis. 1866:165832. doi: 10.1016/j.bbadis.2020.165832

Tseng, H. H. L., Vong, C. T., Kwan, Y. W., Lee, S. M., and Hoi, M. P. M. (2017). Lysosomal $\mathrm{Ca}(2+)$ Signaling Regulates High Glucose-Mediated Interleukin$1 \beta$ Secretion via Transcription Factor EB in Human Monocytic Cells. Front. Immunol. 8:1161.

Tsunemi, T., Ashe, T. D., Morrison, B. E., Soriano, K. R., Au, J., Roque, R. A., et al. (2012). PGC-1 $\alpha$ rescues Huntington's disease proteotoxicity by preventing oxidative stress and promoting TFEB function. Sci. Transl. Med. 4:142ra197.

van Veelen, W., Korsse, S. E., van de Laar, L., and Peppelenbosch, M. P. (2011). The long and winding road to rational treatment of cancer associated with LKB1/AMPK/TSC/mTORC1 signaling. Oncogene 30, 2289-2303. doi: 10.1038/ onc. 2010.630

Vega-Rubin-de-Celis, S., Peña-Llopis, S., Konda, M., and Brugarolas, J. (2017). Multistep regulation of TFEB by MTORC1. Autophagy 13, 464-472. doi: 10 . 1080/15548627.2016.1271514

Visvikis, O., Ihuegbu, N., Labed, S. A., Luhachack, L. G., Alves, A. F., Wollenberg, A. C., et al. (2014). Innate host defense requires TFEB-mediated transcription of cytoprotective and antimicrobial genes. Immunity 40, 896-909. doi: 10.1016/ j.immuni.2014.05.002

Vringer, E., and Tait, S. W. G. (2019). Mitochondria and Inflammation: Cell Death Heats Up. Front. Cell Dev. Biol. 7:100.

Wang, H., Wang, R., Xu, S., and Lakshmana, M. K. (2016). Transcription Factor EB Is Selectively Reduced in the Nuclear Fractions of Alzheimer's and Amyotrophic Lateral Sclerosis Brains. Neurosci. J. 2016:4732837.

Wang, S., Chen, Y., Li, X., Zhang, W., Liu, Z., Wu, M., et al. (2020). Emerging role of transcription factor $\mathrm{EB}$ in mitochondrial quality control. Biomed. Pharmacother. 128:110272. doi: 10.1016/j.biopha.2020.110272

Wang, S., Ni, H. M., Chao, X., Wang, H., Bridges, B., Kumer, S., et al. (2019). Impaired TFEB-mediated lysosomal biogenesis promotes the development of pancreatitis in mice and is associated with human pancreatitis. Autophagy 15, 1954-1969. doi: 10.1080/15548627.2019.1596486

Wang, Y., Huang, Y., Liu, J., Zhang, J., Xu, M., You, Z., et al. (2020). Acetyltransferase GCN5 regulates autophagy and lysosome biogenesis by targeting TFEB. EMBO Rep. 21:e48335.

Wang, Z., and Li, C. (2020). Xenophagy in innate immunity: A battle between host and pathogen. Dev. Comp. Immunol. 109:103693. doi: 10.1016/j.dci.2020. 103693

Willett, R., Martina, J. A., Zewe, J. P., Wills, R., Hammond, G. R. V., and Puertollano, R. (2017). TFEB regulates lysosomal positioning by modulating TMEM55B expression and JIP4 recruitment to lysosomes. Nat. Comm. 8:1580.

Xu, Y., Du, S., Marsh, J. A., Horie, K., Sato, C., Ballabio, A., et al. (2020). TFEB regulates lysosomal exocytosis of tau and its loss of function exacerbates tau pathology and spreading. Mol. Psychiatry 2020:738.

Xu, Y., Ren, J., He, X., Chen, H., Wei, T., and Feng, W. (2019). YWHA/143-3 proteins recognize phosphorylated TFEB by a noncanonical mode for controlling TFEB cytoplasmic localization. Autophagy 15, 1017-1030. doi: 10. 1080/15548627.2019.1569928
You, Y., Bao, W. L., Zhang, S., Li, H. D., Li, H., Dang, W. Z., et al. (2020). Sorting Nexin 10 Mediates Metabolic Reprogramming of Macrophages in Atherosclerosis Through the Lyn-dependent TFEB Signaling Pathway. Circ. Res. 127, 534-549. doi: 10.1161/circresaha.119.315516

Yu, L., Chen, Y., and Tooze, S. A. (2018). Autophagy pathway: Cellular and molecular mechanisms. Autophagy 14, 207-215. doi: 10.1080/15548627.2017. 1378838

Zechner, R., Zimmermann, R., Eichmann, T. O., Kohlwein, S. D., Haemmerle, G., Lass, A., et al. (2012). FAT SIGNALS-lipases and lipolysis in lipid metabolism and signaling. Cell Metab. 15, 279-291. doi: 10.1016/j.cmet.2011.12.018

Zhan, H. Q., Li, S. T., Shu, Y., Liu, M. M., Qin, R., Li, Y. L., et al. (2018). Alpha gene upregulates TFEB expression in renal cell carcinoma with $\mathrm{t}(6 ; 11)$ translocation, which promotes cell canceration. Internat. J. Oncol. 52, 933-944.

Zhang, J., Johnson, J. L., He, J., Napolitano, G., Ramadass, M., Rocca, C., et al. (2017). Cystinosin, the small GTPase Rab11, and the Rab7 effector RILP regulate intracellular trafficking of the chaperone-mediated autophagy receptor LAMP2A. J. Biol. Chem. 292, 10328-10346. doi: 10.1074/jbc.m116.764076

Zhang, X., Cheng, X., Yu, L., Yang, J., Calvo, R., Patnaik, S., et al. (2016). MCOLN1 is a ROS sensor in lysosomes that regulates autophagy. Nat. Commun. 7:12109.

Zhang, Z., Yan, J., Bowman, A. B., Bryan, M. R., Singh, R., and Aschner, M. (2019). Dysregulation of TFEB contributes to manganese-induced autophagic failure and mitochondrial dysfunction in astrocytes. Autophagy 2019, 1-18.

Zhang, Z., Yan, J., Bowman, A. B., Bryan, M. R., Singh, R., and Aschner, M. (2020). Dysregulation of TFEB contributes to manganese-induced autophagic failure and mitochondrial dysfunction in astrocytes. Autophagy 16, 1506-1523. doi: 10.1080/15548627.2019.1688488

Zhao, G. Q., Zhao, Q., Zhou, X., Mattei, M. G., and de Crombrugghe, B. (1993). TFEC, a basic helix-loop-helix protein, forms heterodimers with TFE3 and inhibits TFE3-dependent transcription activation. Mol. Cell Biol. 13, 45054512. doi: 10.1128/mcb.13.8.4505-4512.1993

Zheng, G., Zhan, Y., Li, X., Pan, Z., Zheng, F., Zhang, Z., et al. (2018). TFEB, a potential therapeutic target for osteoarthritis via autophagy regulation. Cell Death Dis. 9:858.

Zhou, X., Yang, J., Zhou, M., Zhang, Y., Liu, Y., Hou, P., et al. (2019). Resveratrol attenuates endothelial oxidative injury by inducing autophagy via the activation of transcription factor EB. Nutr. Metab. 16:42.

Zhu, S. Y., Yao, R. Q., Li, Y. X., Zhao, P. Y., Ren, C., Du, X. H., et al. (2020). Lysosomal quality control of cell fate: a novel therapeutic target for human diseases. Cell Death Dis. 11:817.

Conflict of Interest: The authors declare that the research was conducted in the absence of any commercial or financial relationships that could be construed as a potential conflict of interest.

Publisher's Note: All claims expressed in this article are solely those of the authors and do not necessarily represent those of their affiliated organizations, or those of the publisher, the editors and the reviewers. Any product that may be evaluated in this article, or claim that may be made by its manufacturer, is not guaranteed or endorsed by the publisher.

Copyright (c) 2021 Zhu, Yao, Li, Zhao, Ren, Du and Yao. This is an open-access article distributed under the terms of the Creative Commons Attribution License (CC BY). The use, distribution or reproduction in other forums is permitted, provided the original author(s) and the copyright owner(s) are credited and that the original publication in this journal is cited, in accordance with accepted academic practice. No use, distribution or reproduction is permitted which does not comply with these terms. 